\title{
Refractory thrombocytopenia in SLE patient with massive thigh hematoma and hematuria successfully treated with Rituximab: Case Report
}

Background: Systemic Lupus Erythematosus is a multisystemic inflammatory disease with broad clinical presentation, $9.5 \%$ to $44.5 \%$ of patients with SLE have thrombocytopenia complication of different grades. No significant increase in risk of hemorrhage was found in patients with SLE with moderate thrombocytopenia, while those with severe thrombocytopenia frequently developed bleeding, which is associated with the activity of the disease and potentially life-threatening condition, if left untreated.

Case presentation: We report a case of 35-year-old SLE male patient, presented with sudden painful large non-traumatic ecchymotic lesion on the right thigh extending to calf with macroscopic hematuria. At presentation, platelets count was $50 \times 10^{9} / \mathrm{L}$ with hemoglobin dropping from 10.3 to $6.9 \mathrm{~g} / \mathrm{dl}$. Anticardiolipin antibody and Antiphospholipid antibody were absent. Other factors including infection and visceral involvement were excluded. MRI of the thigh showed huge intramuscular hematoma extending to calf. Satisfactory control was partially achieved with platelet transfusion, pulse steroid, cellcept, IVIG (intravenous immunoglobulins) and consequently Rituximab. Our case describes refractory thrombocytopenia not responding to multiple agents including high dose steroids, cellcept, platelet transfusion, IVIG (intravenous immunoglobulins) and cyklokapron; however, it achieved its complete remission with Rituximab. Bleeding is not usual presentation with moderate thrombocytopenia in SLE patients and presumably played a role in prognosis.

Conclusion: Our case report has demonstrated the efficacy of treating refractory thrombocytopenia resistant to steroids due to Systemic Lupus Erythematosus in whom other reasonable options have been exhausted.

Keywords: moderate thrombocytopenia • systemic lupus erythematosus $•$ rituximab

\section{Introduction}

Thrombocytopenia is a frequent hematologic manifestation in patients with systemic lupus erythematosus, SLE. However, bleeding is not usual manifestation encountered with moderate thrombocytopenia in SLE patient. SLE associated with thrombocytopenia is usually treated with Glucocorticoids, immunosuppressants such as Cyclophosphamide, intravenous immunoglobulin (IVIG) or splenectomy [1]. Rituximab an anti-CD20 a monoclonal antibody that destroys B-cells has shown to be effective in refractory thrombocytopenia in patients with SLE presenting with ecchymosis and hematuria as demonstrated in the following case report [2].

\section{Case presentation}

We describe a 35-year-old male known case of systemic lupus erythematosus diagnosed in 2011, with a history of right lung decortication due to empyema and lupus nephritis in 2011. Presented to the ER with painless gross hematuria and sudden onset of non- traumatic right thigh pain, swelling and ecchymosis extending to the calf (Figure 1). Doppler US was done which showed negative deep venous thrombosis and ill-defined intramuscular hematoma. CBC was done which showed moderate thrombocytopenia $50 \mathrm{x}$ $10^{9} / \mathrm{L}$ and hemoglobin level of $10.3 \mathrm{~g} / \mathrm{dl}$.

Coagulation profile was within normal range. Anticardiolipin antibody and antiphospholipid antibody were absent. Coombs test was positive as well as low C3 and C4 levels. Other factors including infection, fever, visceral involvement and medications such as vancomycin, Amphotericin B and heparin were excluded. MRI was done which showed extensive edema of the right adductor muscle likely due to severe strain with large hematoma measuring $15 \times 6.5 \times 5.3 \mathrm{~cm}$ indenting the adductor muscle group and right semimembranosus muscle. Patient was started on cyklokapron, pulse steroid, 6 units of platelet transfusion and IVIG (intravenous Immunoglobulins).

On the third day, his hemoglobin dropped from 10.3

\author{
Maha J Aljasser, Hanady \\ Manasfi*, Amjad Abdelnabi \& \\ Hanan Y Abdurabu \\ Division of Rheumatology -Specialized \\ Medical Center Hospital, Riyadh, KSA, \\ Saudi Arabia \\ *Author for correspondence: \\ dr_hanadymanasfi@hotmail.com
}




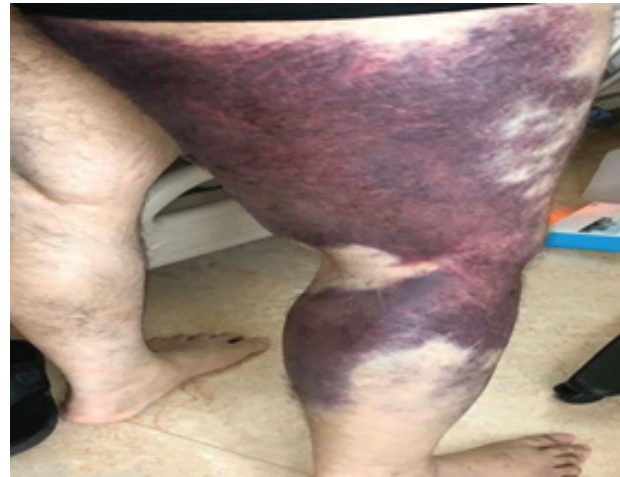

Figure 1. ER with painless gross hematuria and sudden onset of non-traumatic right thigh pain, swelling and ecchymosis extending to the calf.

to $6 \mathrm{~g} / \mathrm{dl}$ and was managed with 2 units of packed RBC. Patient finished pulse steroid with slight improvement in platelet count reaching to $74 \times 10^{9} / \mathrm{L}$. He was then switched to oral steroids $60 \mathrm{mg}$ once daily with platelet count of $80 \times 10^{9} / \mathrm{L}$. Furthermore, his platelet count was fluctuating; he was prescribed two doses of Rituximab, administered two weeks apart because of refractory thrombocytopenia not responding to steroids and other conventional immunosuppressive drugs.

Consequently, three weeks after commencing Rituximab his platelet count reached $120 \times 10^{9} / \mathrm{L}$ upon discharge and on the fourth week, his platelet count had normalized to $200 \times 10^{9} / \mathrm{L}$.

\section{Discussion}

SLE is an autoimmune disease in which the immune system of the body mistakenly attacks different body organs. Around $(9.5 \%$ to $44.5 \%)$ of patients with SLE develop thrombocytopenia [3]. Moreover, in $16 \%$ of patients it can predate the diagnosis of SLE and be the initial manifestation of the disease [4]. Increased destruction of peripheral platelets due to the presence of anti-thrombocyte antibodies on the surface of platelets is the most likely pathologic mechanism [4]. However, the occurrence of spontaneous ecchymotic bleeding in SLE is rare in the presence of moderate thrombocytopenia as seen in our case. On the other hand, those with severe thrombocytopenia will frequently develop bleeding, which is associated with the activity of the disease. SLE-associated thrombocytopenia is usually treated with glucocorticoids, immunosuppressants such as intravenous immunoglobulin (IVIG), Cyclophosphamide and Azathioprine or splenectomy [1]. If thrombocytopenia shows a poor response to these conventional therapies, monoclonal antibodies can be used to normalize platelet count. To the best of our knowledge, a literature search found no case reports of a similar presentation: patient with moderate refractory thrombocytopenia presenting with large thigh hematoma that was managed successfully with Rituximab.

A six-month follow up revealed persistently normal platelet count with significant tapering of steroid to $5 \mathrm{mg}$ daily, Plaquenil and Cellcept tablet were continued.

\section{Conclusion}

We demonstrate a patient who is a known case of SLE presented with huge spontaneous right lower extremity bleeding that had developed with moderate thrombocytopenia, which is not frequently seen in patients with SLE with thrombocytopenia. Despite administering various modalities of treatment such as immunosuppressive agents (i.e cellcept), platelet transfusion pulse steroid and IVIG (intravenous immunoglobulins), Rituximab was required to maintain the platelet count. Rituximab is a chimeric monoclonal anti-CD20 antibody that destroys B- lymphocytes [5]. It is an emerging alternative therapeutic drug for the treatment of various autoimmune diseases [5], including SLE.

In conclusion, our case report has demonstrated the efficacy of treating refractory thrombocytopenia resistant to steroids due to systemic lupus erythematosus in whom other reasonable options have been exhausted. Rituximab administration helped in steroid sparing with satisfactory improvement in platelet count in the treatment of thrombocytopenia secondary to SLE.

\section{Acknowledgment}

The authors have declared no conflicts of interest regarding the publication of this paper.

\section{References}

1. Chang HK. Successful treatment of refractory thrombocytopenia with mycophenolate mofetil in a patient with systemic lupus erythematosus. J. Korean Med. Sci. 20(5), 883-885 (2005)

2. Edwards JCW, Cambridge G. B-cell targeting in rheumatoid arthritis and other autoimmune diseases. Nat. Rev. Immunol. 6(5), 394-403 (2006).

3. Li J, Pan Z, Liu H et al. Retrospective analysis of the risk of hemorrhage associated with moderate and severe thrombocytopenia of 173 patients with systemic lupus erythematosus. Medicine (Baltimore). 97(27), e11356 (2018).

4. Hepburn AL, Narat S, Mason JC. The management of peripheral blood cytopenias in the systemic lupus erythematosus. Rheumatology. 49(12), 2243-2254 (2010).

5. Sabahi R, Anolik JH. B-cell-targeted therapy for systemic lupus erythematosus. Drugs. 66(15), 19331948 (2006) 